\title{
Effets des traitements (chauffage et fermentation par Rhizopus oligosporus sp-T3) de la graine de lupin blanc doux sur certains facteurs de son utilisation nutritionnelle
}

\author{
A Chango, HM Bau, C Villaume *, A Schwertz, \\ JP Nicolas, L Mejean
}

INSERM U-308, 38, rue Lionnois F-54000 Nancy, France

(Reçu le 25 mai 1992; accepté le 17 novembre 1992)

\begin{abstract}
Résumé - Afin d'améliorer la valeur nutritionnelle et les propriétés fonctionnelles du lupin blanc doux (LBD), un procédé de fermentation à l'aide de Rhizopus oligosporus a été étudié.

Une comparaison des effets du trempage suivi de chauffage et de la fermentation a été faite à partir de 3 produits : les graines de lupin non traitées (LBDnt), le lupin chauffé à la vapeur à $100^{\circ} \mathrm{C}$ pendant $30 \mathrm{~min}$. (LBDc) et le lupin fermenté (LBDf) à partir de LBDc, sur les caractéristiques chimiques et le coefficient d'efficacité protéique (CEP).

L'effet majeur du trempage + chauffage consiste en une perte de solubilité des protéines et de la lysine disponible (de $3,02 \pm 0,18 \mathrm{~g} / 16 \mathrm{~g} \mathrm{~N}$ à $2,30 \pm 0,25$ ). La fermentation conduit à une modification de la composition en acides aminés et à une augmentation de la lysine disponible par rapport au chauffage $(2,90 \pm 0,12 \mathrm{~g} / 16 \mathrm{~g} \mathrm{~N})$. La valeur nutritionnelle du lupin non traité est faible (CEP $=0,83 \pm$ $0,09)$. Le traitement thermique la décroit $(0,58 \pm 0,18)$ et la fermentation compense l'effet du chauffage $(0,74 \pm 0,25)$. Ceci suggère que les modifications de la valeur nutritionnelle dépendent plutôt de la disponibilité de la lysine que des variations des taux des autres acides aminés.
\end{abstract}

Lupin / Rhizopus oligosporus / prise alimentaire / CEP / acides aminés

Summary - Effects of the heating and fermentation (by Rhizopus oligosporus (spT3) treatments on the nutritional use of sweet white lupin seeds. For improving the nutritional value and functional properties of sweet white lupin (SWL), a solid fermentation process using Rhizopus oligosporus was developed for the preparation of lupin products.

A comparison of the effects of soaking + thermal treatment and fermentation was made from 3 lupin products: non-treated lupin seeds (SWLnt), heated lupin seeds (steamed at $100^{\circ} \mathrm{C}$ for $30 \mathrm{~min}$ ) $(S W L h)$, and fermented lupin products (SWLf) from SWLh on chemical characteristics and protein efficiency ratio (PER) in rats.

The major effect of soaking and thermal treatment is a loss of protein solubility and available lysine (from $3.02 \pm 0.18 \mathrm{~g} / 16 \mathrm{~g} \mathrm{~N}$ to $2.3 \pm 0.25$ ). Fermentation leads to a modification, of amino acid com-

\footnotetext{
* Correspondance et tirés à part
} 
position and to an increase in available lysine compared to heating $(2.90 \pm 0.12 \mathrm{~g} / 16 \mathrm{~g} \mathrm{~N})$. The nutritional quality of SWLnt is low (PER $=0.83 \pm 0.09$ ), thermal treatment decreases it $(0.58 \pm 0.18$ ) and fermentation compensates heating effect $(0.74 \pm 0.25)$. It is suggested that the modifications of nutritional quality depends on the availability of lysine rather than on the variations of the levels of other amino acids.

\section{lupin / Rhizopus oligosporus / food intake / PER / amino acid}

\section{INTRODUCTION}

La teneur élevée en protéines de $30 \%$ à $51,5 \%$ (Rahma et Rao, 1984) du lupin suggère son emploi comme source potentielle de protéines végétales pour l'alimentation animale et humaine. Les protéines de lupin sont de mieux en mieux connues et sont présentées comme une des plus importantes sources de protéines, en particulier les variétés douces à très faible taux d'alcaloïdes qui sont responsables de l'amertume des graines de lupin et de leur farine. Le lupin existe sous forme de nombreuses espèces (mutabilis, angustifolis, luteus, albus, termis, pour les plus étudiées), présentant des caractéristiques différentes et des variations inter- et intraspécifiques.

La fermentation par une moisissure comme Rhizopus oligosporus utilisée couramment en Extrême-Orient pour la préparation de tempeh (graines de soja cuites et fermentées) est capable d'améliorer la digestibilité et la valeur biologique des produits obtenus en raison de la synthèse de nombreuses enzymes et vitamines et de l'élimination de facteurs antinutritionnels (Kao et Robinson, 1978; Lin, 1980; Truesdell et al, 1987; Sutardi et Buckle, 1988; Bau et al, 1992).

La valeur nutritionnelle des protéines de lupin a été étudiée par de nombreux auteurs (Hill, 1977; Ballester et al, 1980; Schoeneberger et al, 1982; Jecsai et al, 1989). Elle a été trouvée généralement faible par rapport à la caséine et aux protéines de soja chauffées, malgré la bonne digestibilité des protéines. Les facteurs incriminés sont généralement les acides aminés soufrés. Une correction de la valeur nutritionnelle a été obtenue par une supplémentation de ces protéines en méthionine. Ballester et al (1980) expliquent la faible valeur de CEP obtenue avec $L$ albus et $L$ luteus par l'existence possible de structures protéiques impliquant la cystine, lesquelles seraient résistantes aux enzymes, affectant ainsi la disponibilité de cet acide aminé.

Le but de ce travail est d'étudier dans un premier temps l'effet d'un traitement simple de trempage suivi d'un chauffage des graines sur la valeur nutritionnelle de leurs protéines et dans un second temps, l'effet d'un traitement complexe de fermentation suivi d'un chauffage de ces graines selon le procédé de fabrication traditionnellement appliqué au soja. Ce traitement de fermentation est susceptible de provoquer une prédigestion des protéines grâce aux enzymes synthétisées par la souche et pourrait modifier la composition des acides aminés libres en suppléant à la carence en certains acides aminés indispensables et améliorer les caractéristiques organoleptiques (Gauthier et al, 1989).

\section{MATÉRIELS ET MÉTHODES}

Les graines de lupin blanc doux (LBD) variété Lucky, proviennent de la coopérative agricole de Vienne (France). Les farines de graines non traitées (LBDnt) sont obtenues directement par broyage de graines entières avec un broyeur Vertec. Le produit moulu est tamisé avec un tamis AFNOR de $0,63 \mathrm{~mm}$ de maille. 
Les échantillons traités sont obtenus à partir de graines entières, trempées une nuit (16 h), égouttées, stérilisées par chauffage à la vapeur $\left(100^{\circ} \mathrm{C}, 30 \mathrm{~min}\right)$; une fraction des graines est séchée à l'étuve à $50^{\circ} \mathrm{C}$ pendant $24 \mathrm{~h}$ avant d'être réduite en farine (fraction LBDC). La deuxième fraction est inoculée avec des spores de $R$ oligosporus sp T3. L'incubation est faite à l'étuve à $30^{\circ} \mathrm{C}$ pendant $24 \mathrm{~h}$. La fermentation est arrêtée par un second traitement thermique (100 $\left.{ }^{\circ} \mathrm{C}, 30 \mathrm{~min}\right)$. Les graines fermentées sont séchées à l'étuve à $50^{\circ} \mathrm{C}, 24 \mathrm{~h}$ avant d'être broyées puis tamisées (fraction LBDf).

La souche de Rhizopus oligosporus sp-T3 a été gracieusement fournie par le Pr Lin (Institute for Microbial Research, Taïchung, Taiwan).

L'humidité des farines est déterminée par séchage à $105^{\circ} \mathrm{C}$ de l'échantillon moulu, jusqu'au poids constant. Les dosages de l'azote, des cendres et des lipides sont effectués selon les méthodes AOAC (1980). Le taux de protéines totales est le produit du taux d'azote par le facteur 6,25. La solubilité de l'azote est déterminée après dispersion d'1 $\mathrm{g}$ de farine dans $50 \mathrm{ml}$ d'eau distillée ou de $\mathrm{KOH} 0,2 \%$. La suspension est homogénéisée $30 \mathrm{~min}$, centrifugée à 1500 $\mathrm{g}, 10 \mathrm{~min}$, le surnageant filtré sur filtre Whatman $n^{\circ} 4$, une aliquote du filtrat prélevée pour le dosage d'azote. L'azote non protéique (ANP) est déterminé dans un filtrat trichloroacétique suivant la technique décrite par Becker et al (1940). Les glucides éthanolosolubles sont extraits dans l'éthanol à $80 \%$ et dosés suivant la méthode à l'anthrone (Roe, 1955). Le taux d'alcaloides a été déterminé par modification de la méthode colorimétrique décrite par Wink et Hartmann (1981). Une dispersion de $2,5 \mathrm{~g}$ de farine est effectuée dans un volume de $100 \mathrm{ml}$ d'HCl à $0,5 \mathrm{~N}$. Après $1 \mathrm{~h}$ d'agitation, l'homogénat est centrifugé, $10 \mathrm{ml}$ du surnageant sont prélevés puis centrifugés à nouveau à $4000 \mathrm{~g}$ après addition de $2 \mathrm{ml}$ de TCA $3 \mathrm{~N}$. Le surnageant est alors filtré sur filtre Millipore de $0,100 \mu: 3 \mathrm{ml}$ du filtrat sont dosés par spectrophotométrie avec $2 \mathrm{ml}$ de réactif de Reifer modifié ( $14 \mathrm{~g}$ de $\mathrm{KI}, 9 \mathrm{~g}$ de $\mathrm{I}_{2}$ dans $100 \mathrm{ml}$ d'eau), avec pour standard la lupanine. La détermination des phytates a été effectuée suivant la méthode décrite par Latta et Eskin (1980). L'activité antitrypsique, exprimée en unité d'inhibiteur trypsique (UIT) par mg d'échantillon, a été déterminée suivant la méthode de Kakade et al (1974) après extraction à $\mathrm{pH}$ 1. Le taux de lysine disponible a été déterminé par colorimétrie selon la méthode de Carpen- ter (1960), modifiée par Booth (1971). La composition en acides aminés a été déterminée au laboratoire des Agriculteurs de France (Paris), par chromatographie sur colonne échangeuse d'ions, après hydrolyse d'1 $\mathrm{g}$ de farine dans $\mathrm{HCl}$ $6 \mathrm{~N}$ pendant $24 \mathrm{~h}$ à $127^{\circ} \mathrm{C}$. Le tryptophane a été dosé en HPLC-fluorescence avec une colonne C18 de $25 \mathrm{~cm}$, après hydrolyse à la baryte.

La valeur nutritionnelle des protéines a été déterminée sur des rats Wistar mâles (IFFACredo, I'Arbresle, France), âgés de 23 j au début de l'expérimentation dans une animalerie à $23 \pm 1{ }^{\circ} \mathrm{C}, 65-70 \%$ de degré hygrométrique, soumise à une période d'éclairage de 7 à $19 \mathrm{~h}$. A leur arrivée, les rats ont été placés en cages métaboliques individuelles. Après $3 \mathrm{j}$ d'acclimatation avec un régime commercial, 4 lots de 10 rats ont été constitués par tirage au sort. L'homogénéité des lots a été vérifiée. La différence des moyennes du poids des rats de chaque lot ètait inférieure à $5 \mathrm{~g}$. Le régime alimentaire contenait $10 \%$ de protéines (tableau I). L'apport en huile a été ajusté à $9 \%$, en tenant compte du taux de lipides de la farine des graines de départ. La durée de l'expérimentation était de 28 j et la caséine était utilisée comme référence. La prise alimentaire ad libitum individuelle du mé lange farineux était mesurée tous les deux jours et le poids des rats toutes les semaines. Le CEP a èté déterminé selon la procédure de I'AOAC (1970).

Le traitement statistique des résultats a èté réalisé sur Macintosh avec un programme Stat View-ll. Les comparaisons sont faites par une analyse de variance (ANOVA) et un test de Fischer est utilisè pour comparer les différents groupes de régime.

\section{RÉSULTATS}

\section{Composition des graines}

\section{La graine non traitée : (LBDnt)}

Les valeurs de la composition brute des graines de lupin blanc doux sont données dans le tableau II. La teneur en protéines de ces graines qui est de $39 \%$ est impor- 
Tableau I. Composition du régime expérimental.

\begin{tabular}{lr} 
Composition & Pourcentage de régime \\
\hline & \\
Protéines * & $10 \%$ \\
Huile & ajusté à $9 \%$ \\
Cellulose & $5 \%$ \\
Mélange salin ** & $5 \%$ \\
Vitamines $^{* * *}$ & $2 \%$ \\
Amidon qsp **** &
\end{tabular}

\begin{tabular}{|c|c|}
\hline $\mathrm{CaCO}_{3}:$ & $21 \%$ \\
\hline $\mathrm{MgSO}_{4}$ & $9 \%$ \\
\hline $\mathrm{FePO}_{4}, 3 \mathrm{H}_{2} \mathrm{O}$ & $1,47 \%$ \\
\hline $\mathrm{NaCl}:$ & $10,5 \%$ \\
\hline $\mathrm{KAl}\left(\mathrm{SO}_{4}\right)_{2}, 12 \mathrm{H}_{2} \mathrm{O}$ & $0,09 \%$ \\
\hline $\mathrm{KH}_{2} \mathrm{PO}_{4}$ & $31 \%$ \\
\hline $\mathrm{NaF}:$ & $0,057 \%$ \\
\hline $\mathrm{CuSO}_{4}, 5 \mathrm{H}_{2} \mathrm{O}$ & $0,39 \%$ \\
\hline $\mathrm{MnSO}_{4}$ & $0,020 \%$ \\
\hline $\mathrm{KCl}:$ & $12 \%$ \\
\hline $\mathrm{KI}:$ & $0,05 \%$ \\
\hline $\mathrm{Ca}_{3}\left(\mathrm{PO}_{4}\right)_{2}:$ & $14,9 \%$ \\
\hline
\end{tabular}

Vitamines ( $m g / 100 \mathrm{~g}$ de régime) ***

Inositol : $11 \mathrm{mg}$

Niacine : $10 \mathrm{mg}$

Pantothénate de calcium : $6 \mathrm{mg}$

Vitamine A : $2000 \mathrm{UI}$

Chlorure de choline : $166 \mathrm{mg}$

Riboflavine : $2 \mathrm{mg}$

Vitamine B12

Ménadione : $5 \mathrm{mg}$

Chlorhydrate de pyrodoxine : $2 \mathrm{mg}$

Acide folique : $0,2 \mathrm{mg}$

Acide ascorbique : $100 \mathrm{mg}$

Acide $p$-aminobenzoïque : $11 \mathrm{mg}$

Thiamine : $2 \mathrm{mg}$

Vitamine D : $200 \mathrm{UI}$

Biotine : $0,04 \mathrm{mg}$

\footnotetext{
" De lupin ou de caséine ; "*** Quantité suffisante pour préparer $100 \%$ de régime.
}

tante. Le taux de lipides est relativement faible. Le taux de glucides éthanolosolubles est de $11 \%$. Pour les facteurs antinutritionnels (tableau III) une faible présence d'alcaloïdes est notée, tout comme une faible teneur en phytates ainsi qu'une faible activité des inhibiteurs trypsiques.

Les teneurs en la plupart des acides aminés indispensables du lupin blanc doux sont inférieures à celles nécessaires aux besoins de l'homme (protéine standard $F A O$ ), ainsi qu'à celles du besoin des rats.

La carence porte sur les acides aminés soufrés, la valine, la thréonine, la lysine et le tryptophane.

\section{Effet du trempage + chauffage : (LBDC)}

La farine du lupin blanc doux chauffé (LBDC) présente la même composition brute que celle des graines issues directement du trempage (résultats non reproduits). Par rapport aux graines non traitées, on observe une augmentation non significative du taux de protéines. Cette augmentation s'explique essentiellement par la perte de substances hydrosolubles lors du trempage précédant le chauffage. Les glucides éthanolosolubles diminuent de $37 \%$. Une diminution de $26 \%$ des lipides est également observée. Le chauffage ne présente pas d'effet majeur sur les alcaloïdes et les phytates du lupin (tableau III) : seule une réduction imputable au trempage a été observée. L'azote non protéique (ANP) augmente de façon non significative de 0,61 à $0,65 \%$ après chauffage. L'effet bien connu du chauffage sur les inhibiteurs trypsiques thermolabiles est retrouvé. L'effet de ce premier traitement est surtout manifeste sur la solubilité des protéines qui passe de $45,60 \%$ de protéines à $16,60 \%$ au $\mathrm{pH}$ naturel (tableau IV), ainsi que sur la teneur en lysine disponible des protéines. Le chauffage ne modifie pas la composition en acides aminés. 
Tableau II. Composition brute des échantillons de LBD (en g \% de farine).

\begin{tabular}{|c|c|c|c|c|c|c|}
\hline & Protéines & Lipides ${ }^{1}$ & Glucides $^{2}$ & ANP 3 & Humidité & Cendres \\
\hline LBdnt & $38,80 \pm 0,18^{a}$ & $10,20 \pm 0,40^{c}$ & $11,20 \pm 0,47^{c}$ & $0,61 \pm 0,10$ & $9,06 \pm 0,08^{c}$ & $3,20 \pm 0,18^{a}$ \\
\hline LBDC & $40,50 \pm 0,41^{b}$ & $7,55 \pm 0,75^{b}$ & $7,06 \pm 0,99^{b}$ & $0,65 \pm 0,12$ & $7,21 \pm 0,10^{a}$ & $3,28 \pm 0,22^{a}$ \\
\hline LBDf & $40,50 \pm 0,53^{b}$ & $5,44 \pm 0,58^{a}$ & $2,18 \pm 1,12^{\mathrm{a}}$ & $0,72 \pm 0,12$ & $7,96 \pm 0,26^{\mathrm{b}}$ & $4,03 \pm 0,05^{b}$ \\
\hline
\end{tabular}

${ }^{1}$ Lipides extractibles. ${ }^{2}$ Glucides éthanolosolubles. ${ }^{3}$ Azote non protéique. Deux valeurs dans chaque colonne sont significativement différentes lorsqu'elles n'ont pas de lettre en commun.

Tableau III. Composition en facteurs antinutritionnels du LBD (exprimé par rapport à la farine telle quelle).

\begin{tabular}{cccc}
\hline $\begin{array}{c}\text { Alcaloödes } \\
(g \%)\end{array}$ & $\begin{array}{c}\text { Phytates } \\
(g \%)\end{array}$ & $\begin{array}{c}\text { Inhibiteur } \\
\text { trypsique } \\
\text { UIT/mg }\end{array}$ \\
\hline LBDnt & $0,05 \pm 0,00$ & $0,91 \pm 0,13$ & $1,20 \pm 0,02$ \\
LBDc & $0,02 \pm 0,00$ & $0,63 \pm 0,11$ & $0,20 \pm 0,03$ \\
LBDf & $0,03 \pm 0,00$ & $0,63 \pm 0,05$ & $0,10 \pm 0,07$ \\
\hline
\end{tabular}

\section{Effet de la fermentation du LBDc suivi d'un chauffage : (LBDf)}

Par rapport aux graines non traitées, la fermentation est sans effet sur le taux d'azote total des graines. L'azote non protéique (ANP) augmente également de façon non significative jusque $0,72 \%$ après fermentation. Cette augmentation après fermentation semble cependant très faible par rapport à ce qui est habituellement observé avec le soja (0,5 à $2 \%$ par Nout et Rombouts, 1990). Après action de $R$ oligosporus le taux de glucides éthanolosolubles diminue de $80 \%$. Une di-

Tableau IV. Solubilité des protéines et teneur en lysine disponible (expression en \% de protéines totales).

\begin{tabular}{lcrc}
\hline Milieu de solubilité & LBDnt & LBDc & LBDf \\
\hline Eau distillée & $45,6 \pm 0,09^{\mathrm{a}}$ & $16,6 \pm 0,09^{\mathrm{c}}$ & $19,20 \pm 0,09^{\mathrm{b}}$ \\
$\mathrm{KOH}$ à $0,2 \%$ & $87,8 \pm 0,45^{\mathrm{a}}$ & $28,2 \pm 0,44^{\mathrm{b}}$ & $28,89 \pm 0,90^{\mathrm{b}}$ \\
Lysine disponible & $3,02 \pm 0,18^{\mathrm{a}}$ & $2,30 \pm 0,25^{\mathrm{b}}$ & $2,90 \pm 0,12^{\mathrm{a}}$ \\
(en g/16 g d'azote) & & & \\
\hline
\end{tabular}

Deux valeurs de chaque ligne sont significativement différents au seuil de $5 \%$ lorsqu'elles n'ont pas de lettre en commun. 
minution de $46 \%$ des lipides est également observée. La fermentation ne présente pas d'effet majeur sur les alcaloïdes et les phytates de LBDc (tableau III). La cystine, la valine ainsi que la lysine semblent particulièrement affectées parmi les acides aminés indispensables (tableau V). Alors que la teneur en lysine totale diminue après fermentation, on observe en contrepartie une augmentation du taux de la lysine disponible. La solubilité des protéines en milieu aqueux augmente significativement par rapport à celle observée après chauffage tout en restant loin de la valeur obtenue avec l'échantillon non traité.

Tableau V. Composition en acides aminés des échantillons (exprimée en g pour $16 \mathrm{~g}$ d'azote).

AA LBDnt $L B D C \quad L B D f R R^{*}, * * F A O^{* *}$

\begin{tabular}{lrrrrr} 
& & & & & \\
Asp & 10,86 & 10,46 & 9,74 & & \\
Thr & 3,48 & 3,49 & 3,26 & 4,2 & 4,0 \\
Ser & 4,80 & 5,00 & 4,40 & & \\
Glu & 18,53 & 18,82 & 16,91 & & \\
Pro & 3,72 & 3,73 & 4,05 & & \\
Gly & 3,69 & 3,64 & 3,48 & & \\
Ala & 3,12 & 3,14 & 3,02 & & \\
Val & 3,95 & 3,49 & 3,34 & 5,0 & 5,0 \\
Cys & 1,48 & 1,38 & 0,67 & & \\
Met & 0,72 & 0,72 & 0,89 & & \\
Cys + Met & 2,20 & 2,10 & 1,56 & 5,0 & 3,5 \\
lle & 3,74 & 3,88 & 3,71 & 4,6 & 4,0 \\
Leu & 6,58 & 6,92 & 6,65 & 6,2 & 7,0 \\
Tyr & 4,08 & 4,15 & 3,83 & & \\
Phe & 3,87 & 3,86 & 4,37 & & \\
Tyr + Phe & 7,95 & 8,01 & 8,20 & 6,7 & 6,0 \\
His & 2,18 & 2,17 & 2,25 & 2,5 & 1,4 \\
Lys & 4,65 & 4,45 & 4,30 & 7,5 & 5,5 \\
Arg & 10,94 & 10,91 & 8,90 & 5,0 & $\ldots$. \\
Trp & 0,70 & 0,71 & 0,67 & 1,25 & 1,0 \\
& & & & & \\
\hline
\end{tabular}

" Besoin des rats en acides aminés; "* Walker 1983.

\section{Efficacité protéique et prise alimentaire}

Le gain de poids des rats après 28 j de régime à base de LBDnt est très faible et représente $1 / 5$ de celui des rats ingérant le régime caséine (tableau VI). La prise quotidienne de protéines de LBDnt est de $0,74 \mathrm{~g}$, le CEP est égal à 0,83 par rapport à 2,5 pour la caséine. Après chauffage, la prise alimentaire diminue peu, mais le gain de poids et le CEP diminuent significativement tandis que la fermentation provoque une augmentation des 3 paramètres qui demeurent cependant inférieurs à ceux des rats ingérant le régime LBDnt.

\section{DISCUSSION}

Le taux élevé en protéines du lupin blanc doux et sa faible teneur en alcaloïdes expliquent l'intérêt du LBD pour l'alimentation animale et humaine. Les teneurs en protéines du lupin blanc varient de 34,3 à $44,9 \%$ (Hill, 1977). Les $38,8 \%$ de notre échantillon appartiennent à cette gamme. Cependant ces protéines sont carencées en de nombreux acides aminés. Un procédé tel que la fermentation par Rhizopus oligosporus pourrait permettre de modifier la composition en acides aminés.

Traditionnellement, l'étape de fermentation pour la fabrication de tempeh est précédée d'un traitement thermique des graines. Le chauffage à la vapeur $(30 \mathrm{~min}$ à $100^{\circ} \mathrm{C}$ ) ou dans de l'eau bouillante (2$3 \mathrm{~h}$ ) induit une meilleure pénétration de la chaleur et la dénaturation partielle des protéines, facilitant par voie de conséquence la pénétration du mycélium fongique.

L'étape de trempage + chauffage entraîne des effets bien connus : diminution des taux de glucides solubles, de lipides et de facteurs antinutritionnels sans variation majeure de la composition en acides aminés à part une perte de valine. La solubilité 
Tableau VI. Efficacité protéique et prise alimentaire des échantillons de LBD.

\begin{tabular}{llll}
\hline & Ingéré protéique $(g / j)$ & Gain de poids $g / j$ & CEP 1 \\
\hline & & & \\
LBDnt & $0,74 \pm 0,78^{\mathrm{a}}$ & $0,73 \pm 0,12^{\mathrm{a}}$ & $0,83 \pm 0,09^{\mathrm{a}}$ \\
LBDc & $0,67 \pm 0,64^{\mathrm{a}}$ & $0,47 \pm 0,19^{\mathrm{b}}$ & $0,58 \pm 0,18^{\mathrm{b}}$ \\
LBDf & $0,72 \pm 0,62^{\mathrm{a}}$ & $0,64 \pm 0,24^{\mathrm{a}, \mathrm{b}}$ & $0,74 \pm 0,25^{\mathrm{a}, \mathrm{b}}$ \\
Caséine & $1,37 \pm 1,27^{\mathrm{b}}$ & $3,94 \pm 0,41^{\mathrm{c}}$ & $2,50 \pm 0,13^{\mathrm{c}}$ \\
\hline
\end{tabular}

1 Sur la base de 2,5 pour la caséne. Deux valeurs dans chaque colonne sont significativement différentes au seuil de $5 \%$ lorsqu'elles n'ont pas de lettre en commun.

des protéines est fortement diminuée ainsi que le taux de lysine disponible. Parsons et al (1991) ont montré la relation entre la durée de l'autoclavage, le taux de solubilité des protéines de soja, la prise alimentaire et le gain de poids chez des poussins. Leur analyse montre que la durée de l'autoclavage de 0 à $40 \mathrm{~min}$ à $120^{\circ} \mathrm{C}$, provoque une réduction du taux des protéines solubles de soja de 84 à $36 \%$ dans un milieu potassique à $0,2 \%$.

La fermentation conduit à une nouvelle diminution des glucides éthanolosolubles et des lipides qui sont utilisés classiquement comme substrats pour la croissance du mycélium de Rhizopus oligosporus.

En milieu potassique, il n'y a pas de différences entre le produit chauffé et le produit fermenté. En milieu aqueux, la solubilité des protéines de LBDf augmente de $15 \%$ par rapport à celle de LBDc, augmentation pouvant s'expliquer par l'activité des protéases excrétées par le champignon (Wang et Hesseltine, 1965; Lin, 1980). Les protéases, en dégradant les protéines, produiraient entre autres des acides aminés libres, ainsi que de l'ammoniac (Van Buren et al, 1972), ce qui pourrait expliquer l'augmentation du taux de lysine disponible observé avec LBDf par rapport à LBDc. La composition en acides aminés est modifiée par la fermentation. Ainsi, par rapport au lupin trempé et chauffé, le lupin fermenté perd principalement de la cystéine et de l'arginine, mais en contrepartie son taux de phénylalanine et son taux de méthionine augmentent, ce dernier insuffisamment car le taux de l'ensemble des acides aminés soufrés diminue.

Le lupin blanc doux non traité présente une valeur nutritionnelle faible avec un CEP du tiers de celui de la caséine. II est peu consommé par le rat, bien que contenant peu de substances antinutritionnelles. Ceci est sans doute dû à sa composition en acides aminés très déséquilibrée et aux fortes carences en acides aminés soufrés, lysine disponible, valine et tryptophane. En effet, en présence de régimes carencés en acides aminés indispensables, le rat diminue sa prise alimentaire (Harper et al, 1970).

Contrairement à ce qui est souvent observé avec le soja pour lequel le CEP augmente de 1,1 environ à plus de 2,0 après 20 min de chauffage à $100^{\circ} \mathrm{C}$ (Pearson, 1983) grâce à l'élimination de substances antinutritionnelles thermolabiles, le traitement thermique diminue la qualité nutritionnelle des protéines de lupin. Cette observation a déjà été précédemment faite par Pion et al (1979). Ces derniers ont noté une diminution de la prise alimentaire et de la croissance des rats à la suite du 
chauffage du lupin. Le gain de poids diminue significativement alors que la prise alimentaire est peu modifiée, ce qui pourrait être la traduction d'une mauvaise digestibilité à mettre en relation avec la diminution très importante de la solubilité ainsi qu'avec celle de la lysine disponible, alors que le taux de lysine totale diminue relativement peu. Araba et Dale (1990) dans une étude sur des poussins nourris avec des régimes à faible taux de protéines et carencés en lysine ont montré qu'à partir d'une valeur du taux de protéines solubles inférieure à $70 \%$ en milieu potassique, la qualité protéique diminuait.

Après fermentation, la prise alimentaire, le gain de poids et le CEP augmentent de façon non significative chez les rats nourris à base de LBDc pour retrouver des valeurs comparables à celles obtenues avec le lupin blanc non traité. Alors que la solubilité en milieu potassique reste inchangée par rapport à celle du lupin chauffé, le taux de lysine disponible augmente significativement de $26 \%$ par rapport à sa teneur dans la graine chauffée. II apparaît une corrélation entre l'évolution du taux de lysine disponible et celle du CEP d'un traitement à l'autre.

La fermentation par Rhizopus oligosporus est très utilisée et a été bien étudièe dans le cas du soja. L'intérêt porté au procédé de fermentation réside dans la capacité du champignon à produire des enzymes hydrolytiques actives tels que des carbohydratases, lipases, protéases, phytases, etc (Lin, 1980; Nout et Rombouts, 1990). Grâce à l'activité de ces enzymes, les glucides, lipides, protéines et phytates sont partiellement transformés respectivement en sucres simples, acides gras libres, acides aminés et phosphates très rapidement assimilables. De plus, la fermentation permet d'améliorer la valeur nutritionnelle de l'aliment grâce à la synthèse de vitamines par le microorganisme. Le goût, la texture, les arômes sont renforcés avec élimination des goûts de fève (Gauthier et al, 1989). Par sa composition chimique, le lupin présente les substrats nécessaires au développement et à l'activité métabolique du mycélium. Cependant, il se distingue du soja en tant que substrat de croissance de Rhizopus car les phytates résiduels après trempage + chauffage ne sont pas dégradés par la fermentation et le taux d'azote non protéique formé est très faible. Le manque d'effet de la fermentation pourrait être en rapport avec les conditions opératoires qui, pour être efficaces, doivent favoriser une croissance n'entraînant pas une sporulation trop rapide. Ainsi Paredes-Lopez et Harry (1989) ont montré que la fermentation du haricot ne produisait les améliorations recherchées qu'après $72 \mathrm{~h}$ d'action sur des graines sans cuticule. Dans le cas du lupin, nos conditions étaient les mêmes que pour le soja : graines entières et durée de fermentation de $24 \mathrm{~h}$.

\section{CONCLUSION}

Le lupin blanc doux contient un très faible taux d'alcaloides, de phytates et des traces d'inhibiteurs trypsiques thermolabiles. II est riche en protéines avec une composition en acides aminés non équilibrée. Ces protéines manifestent une grande sensibilité au traitement thermique traduite par la diminution de leur solubilité après chauffage.

Les modifications de la valeur nutritionnelle selon les traitements pourraient être imputées à la plus ou moins grande disponibilité de la lysine plutôt qu'aux variations des autres acides aminés. Durant la fermentation, la souche pourrait soit induire une prédigestion partielle de certains composants des graines, soit apporter par son activité métabolique, de la lysine disponible. Les 2 échantillons traités présentent 
un CEP plus faible que l'échantillon non traité. L'intérêt de la fermentation est faible bien qu'elle permette de compenser certains effets négatifs du trempage + chauffage. Compte tenu de sa teneur protéique, de son fort rendement de production et de l'absence de substances nocives, le lupin blanc doux peut être utilisé tel quel comme ingrédient protéique pour formuler des aliments avec d'autres oléagineux, légumineuses ou céréales destinées à la prévention de la malnutrition protéino-énergétique. Les carences en acides aminés de ses protéines pourraient en faire un modèle d'étude de l'effet des acides aminés sur la prise alimentaire.

\section{REMERCIEMENTS}

Les auteurs remercient $\mathrm{J}$ Pothier (Université de Tours, Laboratoire de pharmacochimie des produits naturels et analogues structuraux) pour la fourniture de lupanine qui nous a servi de standard pour le dosage des alcaloïdes.

\section{RÉFÉRENCES}

AOAC (1970) Official methods of analysis. 11th ed. Assoc of Official Analytical Chemist, Washington, DC

AOAC (1980) Official methods of analysis. 13th ed. Assoc of Official Analytical Chemist, Washington, DC

Araba M, Dale NM (1990) Evaluation of protein solubility as an indicator of overprocessing of soybean meal. Poult Sci 69, 76-83

Ballester D, Yanez E, Garcia R, Erazo S, Lopez F, Haardt E, Cornejo S, Lopez A, Pokniak J, Chichester CO (1980) Chemical composition, value and toxicological evaluation of two species of sweet lupine (Lupinus albus and Lupinus luteus). J Agric Food Chem 28, 402-405

Bau HM, Villaume C, Chandrasiri V, Mejean L, Nicolas JP (1992) Détoxification de substances d'origine végétale. Brevet. France Bull Off Propriété Ind $n^{\circ}$ 92/35 ( $n^{\circ}$ de publication 2654585)
Becker HC, Milner RT, Nagel RH (1940) A method for the determination of non protein nitrogen in soybean meal. Cereal Chem 17, $447-457$

Booth VH (1971) Problems in the determination of FDNB-available lysine. J Sci Food Agric 22, 258-624

Carpenter KJ (1960) The estimation of available lysine in animal protein foods. Biochem $J 77$, 604-610

Gauthier S, De Preneuf J, Rocheman M (1989) Les aliments fermentés à base de soja. In : Microbiologie alimentaire. 2. Eds : Bourgeois CM, Larpent JP. Lavoisier, Paris, 160-174

Harper AE, Benevenga NS, Wohlhueter RM (1970) Effect of ingestion of disproportionate amounts of amino acids. Physiol Rev 50, 428-438

Hill GD (1977) The composition and nutritive value of lupin seed. Nutr Abst Rev B 47, 511-529

Jecsai J, Szelenyi GM, Juhasz B (1989) Studies on protein utilization from alkaloid-free luteus albus seed. Acta Agro Hung 38, 149-158

Kakade ML, Rackis JJ, McGhee JE, Puski G (1974) Determination of trypsin inhibitor activity of soy products: a collaborative analysis of an improved procedure. Cereal Chem 51 , 376-382

Kao C, Robinson RJ (1978) Nutritional aspect of fermented foods from chickpea, horsebean and soybean. Cereal Chem 55, 512-517

Latta M, Eskin M (1980) A simple and rapid colorimetric method for phytate determination. $J$ Agric Food Chem 28, 1313-1315

Lin CF (1980) Preparation of shrimp-flavored tempeh using a thermo-tolerable Rhizopus sp T-3 isolated from Indonesia. In: Proc Oriental Ferment Foods. Food Ind Res Dev Inst Taïchung, Taïwan. 167-178

Nout MJR, Rombouts FM (1990) Recent developments in tempe research. $J$ App/ Bacteriol 69, 609-633

Paredes-Lopez O, Harry Gl (1989) Changes in selected chemical and antinutritional components during tempeh preparation using fresh and hardened common beans. J Food $\mathrm{Sci}$ 54, 968-970

Parsons CM, Hashimoto KJ, Baker DH (1991) Soybean protein solubility in potassium hydroxyde: an in vitro test of in vivo protein quality. J Anim Sci69, 2918-2924 
Pearson AM (1983) Soy proteins. In: Development of food protein. 2. (Hudson BJF, ed) Elsevier Applied Science. New York, London, 67-108

Pion R, Mendles-Pereira E, Prugnaud J (1979) Effect of composition and processing on the nutritive value of some leguminous seeds. $J$ Am Oil Chem Soc 56, 150-153

Rahma EH, Rao NMS (1984) Effect of debittering treatment on the composition and protein components of lupin seed (Lupinus termis) flour. J Agric Food Chem 32, 1026-1030

Roe HJ (1955) The determination of sugar in blood and spinal fluid with anthrone reagent. $J$ Biol Chem 212, 335-343

Schoeneberger H, Gross R, Cremer HD, Elmadfa I (1982) Composition and protein quality of Lupinus mutabilis. J Nutr 112, 70-76

Sutardi, Buckle KA (1988) Characterisation of extra-intracellular phytase from Rhizopus oli- gosporus used in tempeh production. Int $\mathrm{J}$ Food Microbiol 6, 67-79

Truesdell DD, Green NR, Acosta PB (1987) Vitamin B12 activity in miso and tempeh. J Food Sci 52, 493-494

Van Buren JP, Hackler LR, Steinkrauss KH (1972) Solubilization of soybean tempeh constituents during fermentation. Cereal Chem 47, 208-224

Walker A (1983) The estimation of protein quality. In: Development of food protein. 2. (Hudson BJF, ed) Elsevier Applied Science, New York, London, 293-323

Wang HL, Hesseltine CW (1965) Studies on the extracellular proteolytic enzymes of Rhizopus oligosporus. Can J Microb 11, 727-732

Wink M, Hartmann T (1981) Sites of enzymatic synthesis of quinolizidine alkaloids and their accumulation in Lupinus polyphyllus. $Z$ Pflanzenphysiol Bd 102, 337-344 